\title{
Die Macht des Verbrauchers
}

\author{
Die aktuelle Diskussion über genmanipulierte Lebensmittel zeigt einmal mehr, \\ dass sich ein Teil der Verbraucher intensiv mit Produkten und ihrer Herstellung \\ auseinandersetzt. Neben klassischen Kaufentscheidungskriterien wie Preis und \\ Qualität gewinnt seit geraumer Zeit die Frage an Bedeutung, ob man ein \\ Produkt "mit gutem Gewissen" kaufen kann. Der wenig greifbare Begriff \\ mit gutem Gewissen lässt sich durch das philosophische Teilgebiet der \\ Ethik konkretisieren.
}

$\mathrm{V}$ Von Barbara Ries und Norbert Sträter erbraucher sind mächtig. Sie können durch bewusste Boykott- oder Kaufentscheidungen Einfluss auf unternehmenspolitische Entscheidungen nehmen (1). Es erscheint daher sinnvoll, Verbraucher durch Auswertung und Offenlegung von Informationen über Unternehmen zur gezielten Nutzung ihrer Marktmacht anzuregen.

In diesem Zusammenhang stellt sich grundsätzlich die Frage, inwiefern entsprechende Informationen durch so genannte ethische Ratings ausgewertet und offen gelegt werden können. Bisherige wissenschaftliche Untersuchungen befassen sich vorwiegend mit einer Untersuchung aus der Anlegerperspektive. Im vorliegenden Beitrag sollen die Möglichkeiten zur Gestaltung eines ethischen Ratings aus Verbrauchersicht erläutert werden.

\section{- Ethische Ratings}

Unter ethischem oder moralischem Handeln wird heutzutage insbesondere das Verfolgen sozialer, kultureller und ökologischer Ziele verstanden (2). Soziale und kulturelle Ziele betreffen dabei die Auswirkungen unternehmerischen Handelns auf interne und externe Personengruppen. Unter ökologischen Gesichtspunkten beeinflusst ein Unternehmen Luft, Erde, Wasser und die Tierwelt seiner Umgebung.

Zur Bewertung eines Unternehmens nach ethischen Kriterien wird es einem so genannten Rating unterzogen. Im Rahmen dieses Verfahrens wird von einer übergeordneten Institution, der Ratingagentur, ein Ratingurteil als Wert auf einer Skala generiert, der eine Art Gütesiegel für das betrachtete Unternehmen ist. Besonders populär ist im wirtschaftswissenschaftlichen Bereich das finanzwirtschaftliche Rating, das die Meinung der Ratingagenturen über die wirtschaftliche Fähig- keit eines Unternehmens ausdrückt, fällige Zahlungsverpflichtungen vollständig und rechtzeitig zu erfüllen.

Neben finanzwirtschaftlichen Kriterien lässt sich ein Unternehmen auch nach ethischen Kriterien beurteilen. Ein ethisches Rating ist die anhand vergleichbarer Messkriterien bewertende Klassifizierung eines Unternehmens entsprechend der Qualität der Handlungen gegenüber allem, was mittelbar und unmittelbar von den Unternehmensaktivitäten betroffenen ist (3).

Zu Beginn des Ratingprozesses erfolgt die Auswahl und Definition der relevanten ethischen Kriterien, die sich in negative und positive Auswahlkriterien unterteilen lassen. Negative Auswahlkriterien kennzeichnen unternehmerisches Handeln, das in einzelnen Aspekten ethisch nicht korrekt ist und zu einem bewussten Ablehnen der Kaufentscheidung führen kann. Positive Auswahlkriterien kennzeichnen hingegen verantwortungsbewusstes unternehmerisches Handeln, das der Allgemeinheit einen positiven Nutzen beschert.

Die Aufstellung des Kriterienkatalogs sollte dabei von übergreifenden zu immer spezielleren Kriterien erfolgen:

Zur Systematisierung der Qualität der Handlungen wird auf oberster Gliederungsebene die innerhalb

Einen Überblick über alle bisher erschienenen Hefte des Informationsdienstes "Ökologisches Wirtschaften" finden Sie auf unseren Internetseiten unter der Adresse:

\section{http://www.oekom.de}

Wir freven uns auf Ihren Besuch! des Unternehmens agierende Partei identifiziert. Hierbei bietet sich eine Einteilung in die Unternehmensbereiche Management, Beschaffung, Produktion, Absatz sowie Forschung und Entwicklung an. Als von den unternehmerischen Handlungen betroffene Bereiche wurden der sozial-kulturelle und der ökologische Bereich identifiziert. Diese beiden Gebiete werden in der Bewertung getrennt voneinander betrachtet. Auf Grundlage dieser Einteilung werden Subkriterien gebildet.

Zunächst wird bewertet, in welcher Branche das Unternehmen tätig ist. Als sozial positiv sind zum Beispiel Dritte-Welt-Läden zu bewerten, als sozial schädlich demgegenüber Branchen wie die Suchtmittel- oder Rüstungsproduktion. Eine ökologisch positiv zu bewertende Branche ist zum Beispiel die Recycling-, negativ hingegen die Atomindustrie. Darüber hinaus kann die vom Unternehmen vermittelte Firmenphilosophie bewertet werden.

\section{Bewertungsebenen}

Auf der Managementebene werden der Führungsstil und die Transparenz der Unternehmensleitung beurteilt. Dies betrifft vor allem interne Anspruchsgruppen wie Eigentümer oder Mitarbeiter. Ein weiterer großer Bereich ist die Personalabteilung. Ein Unternehmen trägt die Verantwortung für seine Mitarbeiter. Insofern ist zu beurteilen, wie gut und in welchem Ausmaß das Unternehmen sich dieser Verantwortung stellt. Des Weiteren kann das Verhalten gegenüber und die Gleichstellung ganz bestimmter Anspruchsgruppen begutachtet werden, zum Beispiel in Bezug auf Frauen, Ausländer oder Behinderte.

Der Bereich Beschaffung ist für das Zur-Verfügung-Stellen der benötigten Inputfaktoren verantwortlich. Hierbei werden Beschaffungsort, Beschaffungsart und Transport überprïft. Im sozialkulturellen Bereich stellen sich hierbei Fragen wie: Werden Rohstoffe im In- oder Ausland eingekauft? Werden fremde Märkte übervorteilt oder ausgebeutet? Auf der ökologischen Seite gilt es einzuschätzen, ob auf Nachhaltigkeit und Naturverträglichkeit geachtet wird.

In der Produktion werden die beschafften Inputfaktoren zu Absatzgütern verarbeitet. Im sozialkulturellen Bereich kann beispielsweise beurteilt werden, in welchem Land sich die Produktionsanlage befindet und ob bei der Standortwahl die kulturellen Gegebenheiten respektiert wurden. Die Produktion wirft aber in erster Linie ökologische Fragen auf, etwa ob Rohstoffe verschwenderisch eingesetzt werden, ob überflüssige Emissionen die Umwelt belasten und vieles mehr. 
Der Unternehmensbereich Absatz kümmert sich um die Vermarktung und den Verkauf der produzierten Waren. Hier wird analog zur Beschaffung der Transport der Waren begutachtet. Ebenfalls bewertungsrelevant könnte die Art der Werbung sein, etwa ob auf jugendverträgliche Darstellungen geachtet wird.

Die Abteilung Forschung und Entwicklung kann wesentlich zu sozialen und ökologischen Innovationen beitragen und sollte daher gesondert bewertet werden. Neben den allgemeinen Forschungsgrundsätzen wird dabei die Art und Weise der Forschung, beispielsweise ob und in welcher Art Tierversuche eingesetzt werden, beurteilt.

Unter dem Sammelbegriff Sonstiges können alle weiteren bewertungsrelevanten aber noch nicht erfassten Einflussfaktoren abgebildet werden. Ein Unternehmen kann sich zum Beispiel durch ein besonderes Engagement auszeichnen, indem es Forschungspreise ausschreibt oder sich durch eine besondere Informationspolitik hervortun, indem es journalistische Aufklärungsarbeit fördert.

Nach der Definition des Kriterienkatalogs erfolgt die Sammlung der in Bezug auf die als relevant erachteten Kriterien benötigten Informationen. Dies stellt sich als extrem schwierig dar, da im Gegensatz zur Dokumentation finanzieller Daten keine Publikationspflicht für derartige Kriterien existiert. Die Informationssuche muss daher breit gefächert sein. Neben einer direkten Befragung der Unternehmen bietet sich die Auswertung der Jahresabschlüsse und der gegebenenfalls freiwillig erstellten Umweltberichte an.

Es wird überprïft, in welcher Art und in welchem Ausmaß die Auswahlkriterien erfüllt werden. Das Ausmaß der Erfüllung kann anhand von Noten dargestellt werden. Minus sechs kann sehr starke negative Erfüllung, plus sechs sehr starke positive Erfüllung bedeuten. Den einzelnen Kriterien werden nun Gewichte zugewiesen, die in der Summe 1 ergeben müssen. Die Ausprägung eines Kriteriums zwischen minus und plus sechs wird mit dem zugewiesenen Gewicht multipliziert; die Summe aller gewichteten Kriterien stellt das aggregierte Ratingurteil dar. Im Rahmen dieses Vorgehens ist minus sechs das schlechteste, plus sechs das beste ethische Urteil.

\section{- Wirkungen und Grenzen ethischer Ratings}

Im Idealfall wirkt sich der Einsatz ethischer Ratings auf die Verbraucher, die bewerteten Unternehmen und den gesamten Markt aus.
Das Rating kann den Informationsbedarf der Verbraucher hinsichtlich der Frage, ob ein Produkt mit ,gutem Gewissen“ gekauft werden kann, decken. Dadurch kann für den Konsumenten ein Zusatznutzen geschaffen werden, indem er eine ganz bewusste und für ihn zufrieden stellende Kaufentscheidung treffen kann.

Das Rating hat außerdem Auswirkungen auf die betroffenen Unternehmen. Durch ihre bewusste Kauf- oder Ablehnungsentscheidung können Konsumenten gezielt schädigendes Unternehmensverhalten sanktionieren und positives Verhalten belohnen. Je größer die Gruppe der Konsumenten ist, die nach diesen Kriterien handeln, desto größer ist der Druck auf die Unternehmen, sich dementsprechend zu verhalten, um ihre Erträge zu sichern oder auszubauen. Die erhöhte Transparenz durch die Bewertung ethischer Kriterien kann somit einerseits nicht erwünschte Einblicke in ein nicht korrektes Verhalten geben aber auch eine Chance sein, sich in diesem Bereich zu profilieren, denn ethisch hochwertige Produkte bieten den Unternehmen die Möglichkeit, auch in stagnierenden Märkten noch steigende Umsätze zu erzielen.

Ganz allgemein können Unternehmen dazu angeregt werden, wichtige Innovationen voran zu bringen, die aus rein ökonomischen Gesichtspunkten zunächst keine Chance auf eine Verwirklichung hätten. Je größer das Gewicht von Ethik-Ratings bei der Kaufentscheidung von Verbrauchern ist, desto größer wird das Interesse der Wirtschaft an einer sozial- und umweltverträglichen Produktion und ebensolchen Produkten sein.

Den Möglichkeiten und Vorzügen ethischer Ratings sind jedoch Grenzen gesetzt. Vor allem das Fehlen einer einheitlichen gesellschaftlichen Moralvorstellung macht ein objektives Ratingurteil unmöglich. Das Urteil hängt insbesondere von der Perspektive des Bewertenden, von der Auswahl der Kriterien und ihrer Gewichtung ab. Je nach einzelner Ausprägung kann es dabei zu ganz unterschiedlichen Ergebnissen kommen. Aus diesen Gründen bleibt das Rating in gewissem Maße immer subjektiv und kann nur einen Ausschnitt, nicht aber die gesamte Realität darstellen.

Die geschilderten Wirkungen auf einzelne Unternehmen oder sogar den gesamten Markt hängen davon ab, ob eine größere Gruppe von Konsumenten die bereitgestellten Informationen wahrnimmt und auch wirklich in disziplinierende Maßnahmen umsetzt. Sollte dies nicht der Fall beziehungsweise die Zielrichtung der Maßnahmen zu heterogen sein, fehlen die für das Unternehmen spürbaren Auswirkungen von Fehl- beziehungsweise vorbildlichem Verhalten und es gäbe keinen Anreiz, sich moralisch einwandfrei zu verhalten.

\section{$>$ Fazit}

Konsumentenreaktionen auf soziale, kulturelle und ökologische Informationen über bestimmte Unternehmen zeigen, dass der Bedarf und die Notwendigkeit einer einheitlichen und vergleichbaren Bewertung bestehen. Es ist daher wünschenswert, dass sich ein einheitliches Qualitätssiegel für die ethische Qualität von Unternehmen herausbildet. Um den unterschiedlichen Präferenzen und Moralvorstellungen der Verbraucher gerecht zu werden ist es notwendig, dass das ethische Ratingurteil nicht nur aus einer verdichteten Kennzahl besteht, sondern zusätzlich ein detaillierter Bericht mit klarer Definition der verwendeten Kriterien geliefert wird. Ob die Kaufkraft der Konsumenten einen Wandel im unternehmerischen Denken herbeiführen kann, können nur künftige Erfahrungen zeigen. Eine Entwicklung in diese Richtung ist sicherlich begrüßenswert.

\section{Anmerkungen}

(1) Vgl. Kleinert, U./ Pentzlin, F.: Ethisches Investment: Instrument zur Durchsetzung sozialer Normen gegenüber Unternehmen? Heidelberg 1994.

(2) Vgl. Hoffmann, J./ Ott, K./ Scherhorn, G.: Ethische Kriterien für die Bewertung von Unternehmen. Frankfurt-Hohenheimer Leiffaden, Frankfurt 1997, S. 16 ff. (3) In Anlehnung an Schneider, H. A.: Ethisches Rating. Frankfurt am Main 2001, S. 254 f., Homolka, W./ NguyenKhac, T.: Ethisch-ökologisches Rating. In: Büschgen, H. E./ Everling, 0. (Hrsg.): Handbuch Rating. Wiesbaden 1996, S. 684 .

\section{Die AutorInnen}

Barbara Ries ist wissenschaftliche Mitarbeiterin am Lehrstuhl für Bank und Finanzwirtschaft der FernUniversität Hagen. Norbert Sträter ist wissenschaftlicher Mitarbeiter am Lehrstuhl für Betriebswirtschaftslehre am Institut für Kreditwesen der Universität Münster. Kontakt: FernUniversität in Hagen, Universitätsstr. 41, 58084 Hagen. Tel. 02331-9872166, E-Mail: barbara.ries@fernuni-hagen.de. Westfälische Wilhelms-Universität Münster, Universitätsstr. 14-16, 48143 Münster. Tel. 0251-8321881,

E-Mail: norbert.straeter@wiwi.uni-muenster.de 
(c) 20I0 Authors; licensee IÖW and oekom verlag. This is an article distributed under the terms of the Creative Commons Attribution Non-Commercial No Derivates License (http://creativecommons.org/licenses/by-nc-nd/3.o/), which permits unrestricted use, distribution, and reproduction in any medium, provided the original work is properly cited. 